\title{
Global well-posedness of solutions to the Cauchy problem of convective Cahn-Hilliard equation \\ Cauchy problem of convective Cahn-Hilliard equation
}

\author{
Xiaopeng Zhao ${ }^{1}$
}

Received: 13 August 2017 / Accepted: 17 January 2018 / Published online: 25 January 2018

(C) Fondazione Annali di Matematica Pura ed Applicata and Springer-Verlag GmbH Germany, part of Springer Nature 2018

\begin{abstract}
In this paper, we consider the global well-posedness of solutions for the Cauchy problem of $N$-dimensional Cahn-Hilliard equation with convective term. We first construct the local smooth solutions; then, by combining some a priori estimates, continuity argument, the local smooth solutions are extended step by step to all $t>0$ provided that the initial data are suitably small and the smooth nonlinear functions satisfy certain local growth conditions at some fixed point $\bar{u} \in \mathbb{R}$. In addition, for the $N$-dimensional Cahn-Hilliard equation without convective term, we also establish the similar result.
\end{abstract}

Keywords Global well-posedness · Convective Cahn-Hilliard equation · Local solution · Cauchy problem

Mathematics Subject Classification $35 \mathrm{~K} 55 \cdot 35 \mathrm{~A} 01 \cdot 35 \mathrm{~B} 45$

\section{Introduction}

The Cahn-Hilliard equation

$$
\left\{\begin{aligned}
\partial_{t} u & =\Delta \mu \\
\mu & :=-\gamma \Delta u+\varphi(u),
\end{aligned}\right.
$$

arises in the study of phase separation on cooling binary solutions such as glasses, alloys and polymer mixtures $[7,25,26]$. Here, $u(x, t)$ is the relative concentration of the two phases, $\Phi(u)=\int_{0}^{u} \varphi(s) \mathrm{d} s$ is a double-well potential with two equal minima at $u^{-} \leq u^{+}$corresponding to the two pure phases, $M$ is the diffusion mobility. During the past years, there are many classical papers related to the study of Cahn-Hilliard equation, see, e.g., Elliott and

Xiaopeng Zhao

zhaoxiaopeng@jiangnan.edu.cn

1 School of Science, Jiangnan University, Wuxi 214122, China 
Zheng [16], Dlotko [12], Temam [30], Gilardi, Miranville and Schimperna [18], Yin [33], Dai and Du [8] and the reference therein.

It is particularly worth mentioning here that Cahn-Hilliard equation is only a phenomenological model. Hence, various modifications of it have been proposed in order to capture the dynamical picture of the phase transition phenomena better: to name only a few, viscous Cahn-Hilliard equation [5,13], Cahn-Hilliard-Gurtin equation [4,22], Cahn-Hilliard-Cook equation [3], Cahn-Hilliard-Brinkman equation [6], Cahn-Hilliard-Hele-Shaw systems [31] and so on.

Recently, many people got interested in the convective Cahn-Hilliard equation

$$
\left\{\begin{aligned}
\partial_{t} u & =\Delta \mu+\nabla \cdot g(u), \\
\mu & :=-\gamma \Delta u+f(u),
\end{aligned}\right.
$$

which arises naturally as a continuous model for the formation of facets and corners in crystal growth $[19,32]$. Here, $u(x, t)$ denotes the slope of the interface. The convective term $\nabla \cdot g(u)[19]$ stems from the effect of kinetic that provides an independent flux of the order parameter, similar to the effect of an external field in spinodal decomposition of a driven system. In [34], Zaks et al. investigated the bifurcations of stations periodic solutions of a convective Cahn-Hilliard equation. Eden and Kalantarov [14,15] established some results on the existence of a compact attractor for the convective Cahn-Hilliard equation with periodic boundary conditions in one space dimension and three space dimension. Very recently, Della Porta and Grasselli [9] considered the initial-boundary value problem of convective nonlocal Cahn-Hilliard equation as dynamical systems and showed that they have bounded absorbing sets and global attractors. In [35], the existence of optimal solutions and optimality condition for 2D convective Cahn-Hilliard equation were studied by Zhao and Liu. Rocca and Sprekels [28] studied a distributed control problem for a 3D convective nonlocal Cahn-Hilliard-type system involving a degenerate mobility and a singular potential.

The goal of this paper is to investigate the global well-posedness for the Cauchy problem of $\mathrm{N}$-dimensional convective Cahn-Hilliard equation

$$
\left\{\begin{array}{l}
\partial_{t} u+\Delta^{2} u-\Delta f(u)-\nabla \cdot g(u)=0, \\
u(x, 0)=u_{0}(x), \quad x \in \mathbb{R}^{N}, \quad t \geq 0 .
\end{array}\right.
$$

As far as we know, there's no reference concerning this aspect of the convective CahnHilliard equation till now. We will prove the existence and uniqueness of global smooth solutions for Cauchy problem (1.1) by using Hoff and Smoller's method [20,21,29] with a slight modification. We show that if the smooth nonlinear functions $f(u)$ and $g(u)$ satisfy certain local growth condition at $u=\bar{u}$ for some $\bar{u} \in \mathbb{R}$ and the $L^{\frac{N(l-1)}{3}}(\mathbb{R})$-norm of the initial data is sufficiently small, then there exists a unique global smooth solution $u(t, x)$ to the Cauchy problem (1.3). More precisely, the result can be stated as follows.

Theorem 1.1 Suppose that $r>0$ and $l>1$ are two given positive constants, $L=\max \{5, N\}$ and $\bar{u} \in \mathbb{R}$ is some fixed constant. Assume that $u_{0}(x)-\bar{u} \in$ $L^{\frac{N(l-1)}{3}}\left(\mathbb{R}^{N}\right) \bigcap L^{\infty}\left(\mathbb{R}^{N}\right)$ with $\left\|u_{0}(x)-\bar{u}\right\|_{L^{\infty}} \leq r$ and $\left\|u_{0}(x)-\bar{u}\right\|_{L^{\frac{N(l-1)}{3}}}$ sufficiently small. The nonlinear functions $f(u), g(u) \in C^{L}(\bar{B}(\bar{u}, 2 r), \mathbb{R})$ satisfying $f(u)=O(1)|u-\bar{u}|^{p}$ and $g(u)=O(1)|u-\bar{u}|^{l}$ as $u \rightarrow \bar{u}$, where $p=\frac{2 l+1}{3}$. Then, there exists a unique global smooth solution $u(t, x) \in L^{\infty}\left(0, \infty ; L^{\frac{N(l-1)}{3}}\left(\mathbb{R}^{N}\right)\right)$ for the Cauchy problem (1.1), which satisfies

$$
\|u(t, x)-\bar{u}\|_{L^{\infty}} \leq 2 r, \quad t \geq 0 .
$$


Remark 1.1 For the details on the fractional Laplacian operator $(-\Delta)^{\gamma}\left(\gamma \in \mathbb{R}^{+}\right)$and fractional Sobolev spaces, the reader may refer to $[1,2,10,23]$.

Remark 1.2 The solution $u(t, x)$ is said to be the global smooth solution to the convective Cahn-Hilliard equation if $u(t, x) \in C^{1,4}([0, \infty) \times \mathbb{R})$.

It is worth pointing that Liu et. al. [24] studied the global well-posedness of Cauchy problem for Cahn-Hilliard equation in $\mathbb{R}^{N}$ :

$$
\left\{\begin{array}{l}
\partial_{t} u+\Delta^{2} u-\Delta f(u)=0, \\
u(x, 0)=u_{0}(x), \quad x \in \mathbb{R}^{N}, \quad t \geq 0 .
\end{array}\right.
$$

The authors supposed that $f(u)=O(1)|u|^{1+\frac{2}{N}}$ and $\|u-\bar{u}\|_{L^{1}}$ sufficiently small, obtained the global well-posedness and decay rate of solutions. In Theorem 1.1, we consider the convective Cahn-Hilliard equation with two nonlinear terms $\Delta f(u)$ and $\nabla \cdot g(u)$. We give a fewer restriction on the nonlinear functions $f(u)$ and $g(u)$, only assume $f(u)=O(1)|u-\bar{u}|^{\frac{2 l+1}{3}}$, $g(u)=O(1)|u-\bar{u}|^{l}$ with $l>1$ and $\|u-\bar{u}\|_{L \frac{N(l-1)}{3}}$ sufficiently small, proved the global well-posedness of solutions. In fact, our method can also be generalized to the classical Cahn-Hilliard equation without convective term.

Theorem 1.2 Suppose that $r>0$ and $l>1$ are two given positive constants, $L=\max \{5, N\}$ and $\bar{u} \in \mathbb{R}$ is some fixed constant. Assume that $u_{0}(x)-\bar{u} \in$ $L^{\frac{N(l-1)}{2}}\left(\mathbb{R}^{N}\right) \bigcap L^{\infty}\left(\mathbb{R}^{N}\right)$ with $\left\|u_{0}(x)-\bar{u}\right\|_{L^{\infty}} \leq r$ and $\left\|u_{0}(x)-\bar{u}\right\|_{L^{\frac{N(l-1)}{2}}}$ sufficiently small. The nonlinear function $f(u) \in C^{L}(\bar{B}(\bar{u}, 2 r), \mathbb{R})$ satisfying $f(u)=O(1)|u-\bar{u}|^{l}$ as $u \rightarrow \bar{u}$. Then, there exists a unique global smooth solution $u(t, x) \in L^{\infty}\left(0, \infty ; L^{\frac{N(l-1)}{2}}\left(\mathbb{R}^{N}\right)\right)$ for the Cauchy problem (1.5), which satisfies

$$
\|u(t, x)-\bar{u}\|_{L^{\infty}} \leq 2 r, \quad t \geq 0 .
$$

Remark 1.3 We assume that $f(u)=O(1)|u-\bar{u}|^{l}(l>1)$ as $u \rightarrow \bar{u}$ in Theorem 1.2. When $l=1+\frac{2}{N}$, we recover the result in [24].

The rest of this paper is organized as follows. In the next section, we introduce some preliminary results. The proof of Theorem 1.1 is postponed in Sect. 3. We also give a brief proof of Theorem 1.2 in the final section of this paper.

Throughout this article, we use $C$ to denote the generic constant that can take different values in different places. In addition, $L^{p}\left(\mathbb{R}^{N}\right)(1 \leq p \leq \infty)$ represents the $N$-dimensional Lebesgue space with norm

$$
\|u\|_{L^{p}}=\left(\int_{\mathbb{R}^{3}}|u(x, t)|^{p} \mathrm{~d} x\right)^{\frac{1}{p}}, \quad\|u\|_{L^{\infty}}=\text { ess } \sup _{x \in \mathbb{R}^{3}}|u(x, t)| .
$$

Moreover, for each $k \in \mathbb{Z}^{+}, D^{k} u$ is used to denote the set of all $k$ th order derivatives of $u(t, x)$ with respect to $x$ and $\left|D^{k} u\right|^{2}=\sum_{|\alpha|=k}\left|\frac{\partial^{|\alpha|} u}{\partial x_{1}^{\alpha_{1} \ldots \partial_{x}}}\right|^{2}$, where $\alpha=\left(\alpha_{1}, \alpha_{2}, \ldots, \alpha_{N}\right)$ is some multi-index and $|\alpha|=\sum_{j=1}^{N} \alpha_{j}$ stands for the length of $\alpha$.

\section{Preliminaries}

The following two lemmas will be used in this section. 
Lemma 2.1 ( [27]) If $1 \leq p \leq r \leq q \leq \infty$ and $u \in L^{p}\left(\mathbb{R}^{N}\right) \bigcap L^{q}\left(\mathbb{R}^{N}\right)$, then $u \in L^{r}\left(\mathbb{R}^{N}\right)$ with

$$
\|u\|_{L^{r}} \leq\|u\|_{L^{p}}^{\alpha}\|u\|_{L^{q}}^{1-\alpha}, \quad \text { where } \frac{1}{r}=\frac{\alpha}{p}+\frac{1-\alpha}{q} .
$$

Lemma 2.2 (Strauss's inequality [29]) Suppose that $M(t)$ is a nonnegative continuous function of t. $M(t)$ satisfies

$$
M(t) \leq d_{1}+d_{2} M(t)^{r}
$$

in some interval containing 0 , where $d_{1}$ and $d_{2}$ are positive constants and $r>1$. If $M(0) \leq d_{1}$ and

$$
d_{1} d_{2}<\left(1-r^{-1}\right) r^{-(r-1)^{-1}},
$$

then in the same interval

$$
M(t) \leq \frac{d_{1}}{1-r^{-1}} .
$$

In the proof of lemmas and theorems, we frequently employ the Gagliardo-Nirenberg inequality:

Lemma 2.3 (Gagliardo-Nirenberg inequality [17]) Let $u \in L^{q}\left(\mathbb{R}^{n}\right), \nabla^{m} u \in L^{r}\left(\mathbb{R}^{n}\right)$, $1 \leq q, r \leq \infty$. Then, there exists a positive constant $C=C(n, m, j, a, q, r)$, such that

$$
\left\|\nabla^{j} u\right\|_{L^{p}} \leq C\left\|\nabla^{m} u\right\|_{L^{r}}^{a}\|u\|_{L^{q}}^{1-a}
$$

where

$$
\frac{1}{p}=\frac{j}{n}+a\left(\frac{1}{r}-\frac{m}{n}\right)+(1-a) \frac{1}{q}, \quad 1 \leq p \leq \infty, 0 \leq j \leq m, \frac{j}{m} \leq a \leq 1 .
$$

We give the $L^{p}\left(\mathbb{R}^{3}, \mathbb{R}\right)$-estimate on the fundamental solution to the convective CahnHilliard equations.

Lemma 2.4 Suppose that $k(t)=\mathcal{F}^{-1}\left(e^{-|\xi|^{4} t}\right)$, where $\xi, x \in \mathbb{R}^{N}$ and $t>0$. We have

$$
\begin{array}{r}
\|k(t)\|_{L^{p}\left(\mathbb{R}^{N}\right)} \leq \frac{c_{p}}{c_{q}} t^{-\frac{N}{4}\left(\frac{1}{q}-\frac{1}{p}\right)}\|k(t)\|_{L^{q}\left(\mathbb{R}^{N}\right)}, \\
\left\|D^{j} k(t)\right\|_{L^{p}\left(\mathbb{R}^{N}\right)} \leq \frac{c_{p, j}}{c_{q}} t^{-\frac{N}{4}\left(\frac{1}{q}-\frac{1}{p}\right)-\frac{j}{4}}\|k(t)\|_{L^{q}\left(\mathbb{R}^{N}\right)}, \quad j=1,2, \ldots,
\end{array}
$$

where $c_{p}, c_{q}$ and $c_{p, j}$ are positive constants with $c_{1}=1$ and $\mathcal{F}^{-1}$ denotes the inverse Fourier transformation with respect to $\xi$.

Proof Let $\xi=\eta t^{-\frac{1}{4}}$. Hence

$$
k(t, x)=\int_{\mathbb{R}^{N}} \mathrm{e}^{-|\xi|^{4} t} \mathrm{e}^{i x \cdot \xi} \mathrm{d} \xi=t^{-\frac{N}{4}} \int_{\mathbb{R}^{N}} \mathrm{e}^{-|\eta|^{4}} \mathrm{e}^{i x \cdot \eta t^{-\frac{1}{4}}} \mathrm{~d} \eta .
$$

Let $G(y)=\int_{\mathbb{R}^{N}} \mathrm{e}^{-|\eta|^{4}} \mathrm{e}^{i y \cdot \eta} \mathrm{d} \eta$. Clearly, $G(y)$ is a rapidly decreasing function. Then

$$
\begin{aligned}
\left(\int_{\mathbb{R}^{N}}|k(t, x)|^{p} \mathrm{~d} x\right)^{\frac{1}{p}} & =t^{-\frac{N}{4}}\left(\int_{\mathbb{R}^{N}} \mid G\left(\left.x t^{-\frac{1}{4}}\right|^{p} \mathrm{~d} x\right)^{\frac{1}{p}}\right. \\
& =t^{-\frac{N}{4}} t^{\frac{N}{4 p}}\left(\int_{\mathbb{R}^{N}}|G(z)|^{p} \mathrm{~d} z\right)^{\frac{1}{p}}=c_{p} t^{-\frac{N}{4}\left(1-\frac{1}{p}\right)} .
\end{aligned}
$$


Similarly, we have

$$
\left(\int_{\mathbb{R}^{N}}|k(t, x)|^{q} \mathrm{~d} x\right)^{\frac{1}{q}}=c_{q} t^{-\frac{N}{4}\left(1-\frac{1}{p}\right)} .
$$

Combining the above two equalities together gives (2.1). In addition,

$$
D^{k} k(t, x)=t^{-\frac{N+k}{\alpha}} D_{y}^{k} G\left(x t^{-\frac{1}{\alpha}}\right) .
$$

By simple calculations, we obtain (2.2). The proof is completed.

\section{Proof of Theorem 1.1}

In order to prove the global existence and uniqueness of smooth solutions for the Cauchy problem of convective Cahn-Hilliard equation, we first state the local existence result.

Lemma 3.1 Suppose that $\max _{u \in \bar{B}(\bar{u}, 2 r)} \sum_{k=1}^{L}\left(\left|D^{k} f(u)\right|+\left|D^{k} g(u)\right|\right)=b$ and the conditions listed in Theorem 1.1 are satisfied. Then, there exists a unique local solution $u(x, t)$ on the strip $\Pi_{t_{1}}:=\left\{(x, t): x \in \mathbb{R}^{N}, 0<t \leq t_{1}\right\}$ such that

$$
\|u(x, t)-\bar{u}\|_{L^{\infty}} \leq 2 r, \quad 0 \leq t \leq t_{1},
$$

where $t_{1}=\min \left\{1, \frac{1}{64 b^{2} c_{1,2}^{2}},\left(\frac{3}{16 b c_{1,1}}\right)^{\frac{4}{3}}\right\}$. In addition, assume that $C_{k}$ is a continuous increasing function of $t-s_{k}^{\prime}$, then for each $0<s_{1}^{\prime}<s_{2}^{\prime}<\cdots<s_{N}^{\prime}<t \leq t_{1}$, we have

$$
\left\|D^{k} u(t, x)\right\|_{L^{\infty}} \leq\left(t-s_{k}^{\prime}\right)^{-\frac{k}{4}} C_{k}\left(r, s_{k}^{\prime}-s_{1}^{\prime}, t-s_{k}^{\prime}\right), \quad k=1,2, \ldots L .
$$

Proof The smooth solution of Cauchy problem (1.3) should satisfy the following integrodifferential equation

$$
\begin{aligned}
u(t, x)= & \int k(t, x-y) u_{0} \mathrm{~d} y-\int_{0}^{t} \mathrm{~d} s \int \Delta k(x-y, t-s) f(u(s, y)) \mathrm{d} y \\
& -\int_{0}^{t} \mathrm{~d} s \int \nabla \cdot k(x-y, t-s) g(u(s, y)) \mathrm{d} y .
\end{aligned}
$$

We first prove that there is a sufficiently small $t_{1}>0$ such that the integro-differential equation (3.3) admits a unique continuous solution $u(t, x)$ on the strip $\Pi_{t_{1}}$. Then, if we can show that the solution obtained above is a smooth solution, such a $u(t, x)$ is indeed a local smooth solution to problem (1.1).

Assume $T(t) u=k(t, x) * u(t, x)$. Hence, (3.3) is equivalent to

$$
u(t, x)=T(t) u_{0}-\int_{0}^{t} \Delta T(t-s) f(u(s)) \mathrm{d} s-\int_{0}^{t} \nabla \cdot T(t-s) g(u(s)) \mathrm{d} s .
$$

It is easy to see that $T(t) 1=1$. In order to prove that there exists a local smooth solution for (3.3), we just need to show that there exists a local smooth solution for

$$
\begin{gathered}
u(t, x)-\bar{u}=T(t)\left(u_{0}-\bar{u}\right)-\int_{0}^{t} \Delta T(t-s) f(u(s)) \mathrm{d} s \\
-\int_{0}^{t} \nabla \cdot T(t-s) g(u(s)) \mathrm{d} s .
\end{gathered}
$$


By using the successive approximations method [11,24]: Set $u^{(0)}(t, x)=u_{0}(x)$ and for $n \geq 1$, we define

$$
\begin{aligned}
u^{(n)}(t, x)-\bar{u}= & T(t)\left(u^{(0)}-\bar{u}\right)-\int_{0}^{t} \Delta T(t-s) f\left(u^{(n-1)}(s)\right) \mathrm{d} s \\
& -\int_{0}^{t} \nabla \cdot T(t-s) g\left(u^{(n-1)}(s)\right) \mathrm{d} s .
\end{aligned}
$$

Obviously, $u^{(n)}(t, x)$ is well defined on $[0, \infty) \times \mathbb{R}$ for each $n \geq 0$.

Set $v^{(n)}(t, x)=u^{(n)}(t, x)-\bar{u}$ and $\mathscr{I} \chi \mathscr{I}=\sup _{(t, x) \in \Pi_{t_{1}}}|\chi(t, x)|$. By induction, we prove that if $t_{1}=\min \left\{1, \frac{1}{64 b^{2} c_{1,2}^{2}},\left(\frac{3}{16 b c_{1,1}}\right)^{\frac{4}{3}}\right\}$, then

$$
\mathbb{I} v^{(n)} \mathbb{I} \leq 2 r .
$$

From the assumption we imposed on the initial data, we obtain (3.7) holds for $n=0$. As to the case $n=1$, since Hausdorff-Young's inequality together with the fact that $u_{0}(x) \in \bar{B}(\bar{u}, r)$ implies that

$$
\left|\int k(t, x-y)\left(u_{0}(y)-\bar{u}\right) \mathrm{d} y\right| \leq\left\|u_{0}-\bar{u}\right\|_{L^{\infty}} \leq r,
$$

we have from (3.6) and (3.8) that

$$
\begin{aligned}
\mathbb{I} v^{(1)}(t, x) \mathbb{I} & \leq r+b c_{1,2} \int_{0}^{t}(t-s)^{-\frac{1}{2}} \mathbb{I} v^{(0)} \mathbb{I} \mathrm{d} s+b c_{1,1} \int_{0}^{t}(t-s)^{-\frac{1}{4}} \mathscr{I} v^{(0)} \mathbb{I} \mathrm{d} s \\
& \leq r+4 r b c_{1,2}\left(t_{1}\right)^{\frac{1}{2}}+\frac{8}{3} r b c_{1,1}\left(t_{1}\right)^{\frac{3}{4}} \leq 2 r .
\end{aligned}
$$

Hence, (3.7) is right for $n=1$. In addition, assume that (3.7) holds for $n \leq m-1$ for some positive integer $m \geq 1$. We now show that (3.7) also holds for $n=m$. Applying Hausdorff-Young's inequality and (3.6), we deduce that

$$
\begin{aligned}
\mathbb{I} v^{(m)} \mathbb{I} & \leq r+b \int_{0}^{t}\|\Delta k(t-s)\|_{L^{1}} \mathbb{I} v^{(m-1)} \mathbb{I} \mathrm{d} s+b \int_{0}^{t}\|\nabla k(t-s)\|_{L^{1}} \mathbb{I} v^{(m-1)} \mathbb{I} \mathrm{d} s \\
& \leq r+b c_{1,2} \mathbb{I} v^{(m-1)} \mathbb{I} \int_{0}^{t}(t-s)^{-\frac{1}{2}} \mathrm{~d} s+b c_{1,1} \Phi v^{(m-1)} \mathbb{I} \int_{0}^{t}(t-s)^{-\frac{1}{4}} \mathrm{~d} s \\
& \leq r+4 r b c_{1,2}\left(t_{1}\right)^{\frac{1}{2}}+\frac{8}{3} r b c_{1,1}\left(t_{1}\right)^{\frac{3}{4}} \leq 2 r .
\end{aligned}
$$

Therefore, (3.7) holds for $n=m$ and by induction, (3.7) is true for any $n \geq 0$.

We also prove that $v^{(n)}(t, x)$ satisfies

$$
\begin{aligned}
\mathscr{I} v^{(n)}-v^{(n-1)} \mathbb{I} & \leq \frac{\left(\mathcal{C}_{1}\left(t_{1}\right)^{\frac{1}{2}}\right)^{n-1}}{\Gamma\left(\frac{n-1}{2}+\frac{1}{2}\right)} M_{1}+\frac{\left(\mathcal{C}_{2}\left(t_{1}\right)^{\frac{3}{4}}\right)^{n-1}}{\Gamma\left(\frac{n-1}{2}+\frac{1}{2}\right)} M_{2} \\
& \leq \frac{\mathcal{C}_{1}^{n-1} M_{1}+\mathcal{C}_{2}^{n-1} M_{2}}{\Gamma\left(\frac{n-1}{2}+\frac{1}{2}\right)}, \quad n \geq 1,
\end{aligned}
$$


where $M_{1}=2 r b c_{1,2} \sqrt{\pi}, \mathcal{C}_{1}=b c_{1,2} \sqrt{\pi}, M_{2}=\frac{3}{4} r b c_{1,1} \sqrt{\pi}$ and $\mathcal{C}_{2}=b c_{1,1} \sqrt{\pi}$. (3.11) can be established by induction. For $n=1$, from (3.6) that

$$
\begin{aligned}
& \mathbb{I} v^{(1)}(t, x)-v^{(0)}(t, x) \mathbb{I} \\
& \leq \int_{0}^{t}\|\Delta k(t-s)\|_{L^{1}} \mathbb{I} f\left(u^{(0)}\right)-f(\bar{u}) \mathbb{I} \mathrm{d} s \\
& \quad+\int_{0}^{t}\|\nabla k(t-s)\|_{L^{1}} \mathbb{I} g\left(u^{(0)}\right)-g(\bar{u}) \mathbb{I} \mathrm{d} s \\
& \leq r b c_{1,2} \int_{0}^{t}(t-s)^{-\frac{1}{2}} \mathrm{~d} s+r b c_{1,1} \int_{0}^{t}(t-s)^{-\frac{1}{4}} \mathrm{~d} s \\
& \leq 2 r b c_{1,2}\left(t_{1}\right)^{\frac{1}{2}}+\frac{4}{3} r b c_{1,1}\left(t_{1}\right)^{\frac{3}{4}} \leq \frac{M_{1}+M_{2}}{\sqrt{\pi}},
\end{aligned}
$$

which implies that the estimate (3.11) is right for $n=1$. Suppose that (3.11) holds for $n \leq m-1$, where $m \geq 2$ is a positive integer. Then, we can get from (3.6) that

$$
\begin{aligned}
& \mathbb{I} v^{(m)}(t, x)-v^{(m-1)}(t, x) \mathbb{I} \\
& \leq \int_{0}^{t}\|\Delta k(t-s)\|_{L^{1}} \mathbb{I} f\left(u^{(m-1)}\right)-f\left(u^{(m-2)}\right) \mathbb{d} \mathrm{d} s \\
& \quad+\int_{0}^{t}\|\nabla k(t-s)\|_{L^{1}} \mathbb{I} g\left(u^{(m-1)}\right)-g\left(u^{(m-2)}\right) \mathbb{d} \mathrm{d} s \\
& \leq b c_{1,2} \int_{0}^{t}(t-s)^{-\frac{1}{2}} \frac{\left(\mathcal{C}_{1} s^{\frac{1}{2}}\right)^{m-2}}{\Gamma\left(\frac{m-2}{2}+\frac{1}{2}\right)} M_{1} \mathrm{~d} s \\
& +b c_{1,1} \int_{0}^{t}(t-s)^{-\frac{3}{4}} \frac{\left(\mathcal{C}_{2} s^{\frac{3}{4}}\right)^{m-2}}{\Gamma\left(\frac{m-2}{2}+\frac{1}{2}\right)} M_{2} \mathrm{~d} s \\
& \leq b c_{1,2} \sqrt{\pi} \frac{\Gamma\left(\frac{m-2}{2}+1\right)}{\Gamma\left(\frac{m-2}{2}+\frac{3}{2}\right)}\left(t_{1}\right)^{\frac{m-1}{2}} \frac{\left(\mathcal{C}_{1}\right)^{m-2} M_{1}}{\Gamma\left(\frac{m-2}{2}+\frac{1}{2}\right)} \\
& \quad+b c_{1,1} \sqrt{\pi} \frac{\Gamma\left(\frac{m-2}{2}+1\right)}{\Gamma\left(\frac{m-2}{2}+\frac{3}{2}\right)}\left(t_{1}\right)^{\frac{3(m-1)}{4}} \frac{\left(\mathcal{C}_{2}\right)^{m-2} M_{2}}{\Gamma\left(\frac{m-2}{2}+\frac{1}{2}\right)} \\
& \leq \frac{\left(\mathcal{C}_{1}\right)^{m-1} M_{1}+\left(\mathcal{C}_{2}\right)^{m-1} M_{2}}{\Gamma\left(\frac{m-2}{2}+\frac{1}{2}\right)},
\end{aligned}
$$

which implies that (3.11) is true for $n=m$. By induction again, we derive that the estimate (3.11) holds for any $n \geq 1$.

It is easy to see that $\sum_{n=0}^{\infty} \frac{\left(\mathcal{C}_{1}\right)^{m-1} M_{1}+\left(\mathcal{C}_{2}\right)^{m-1} M_{2}}{\Gamma\left(\frac{m-2}{2}+\frac{1}{2}\right)}$ is convergent. Then, follows from (3.11), we obtain $v^{(n)}(t, x)$ converges uniformly to $v(t, x)=u(t, x)-\bar{u}$ on the strip $\Pi_{t_{1}}$. It is clear that the unique limit $u(t, x)$ is a continuous solution of (3.3) on $\Pi_{t_{1}}$.

In order to show that such a $u(t, x)$ obtained above is indeed a smooth solution of problem (1.1) on $\Pi_{t_{1}}$, we also need to obtain the regularity of $u(t, x)$. To do so, we only need to derive the following estimates: For each $1 \leq k \leq L, n \geq 1$, there exists a $C_{k}$ which is a continuous increasing function of $t-s_{k}^{\prime}$ such that

$$
\left\|D^{k} u^{(n)}(t)\right\|_{L^{\infty}} \leq\left(t-s_{k}^{\prime}\right)^{-\frac{k}{4}} C_{k}\left(r, s_{k}^{\prime}-s_{1}^{\prime}, t-s_{k}^{\prime}\right), \quad s_{k}^{\prime}<t \leq t_{1} .
$$


By (3.6) and the semigroup property of $T(t)$, we derive that

$$
\begin{aligned}
u^{(n)}(t, x)-\bar{u} & =T(t-\bar{t})\left(u^{(n)}(\bar{t}, x)-\bar{u}\right)-\int_{\bar{t}}^{t} \Delta T(t-s) f\left(u^{(n-1)}(s)\right) \mathrm{d} s \\
& \left.-\int_{\bar{t}}^{t} \nabla \cdot T(t-s) g\left(u^{(n-1}\right)(s)\right) \mathrm{d} s .
\end{aligned}
$$

For $k=1$, we have

$$
\begin{aligned}
D u^{(n)}(t, x)= & D T\left(t-s_{1}^{\prime}\right)\left(u^{(n)}\left(s_{1}^{\prime}, x\right)-\bar{u}\right)-\int_{s_{1}^{\prime}}^{t} D^{3} T(t-s) f\left(u^{(n-1)}(s)\right) \mathrm{d} s \\
& -\int_{s_{1}^{\prime}}^{t} D^{2} T(t-s) g\left(u^{(n-1)}(s)\right) \mathrm{d} s .
\end{aligned}
$$

Applying Hausdorff-Young's inequality, we deduce that

$$
\begin{aligned}
& \mathbb{I}\left[D u^{(n)}(t) \mathbb{I} \leq c_{1,1}\left(t-\bar{s}_{1}^{\prime}\right)^{-\frac{1}{4}} r+b c_{1,5} \int_{a_{1}^{\prime}}^{t}(t-s)^{-\frac{3}{4}} \mathbb{I} v^{(n-1)} \mathbb{I} \mathrm{d} s\right. \\
& \quad+b c_{1,2} \int_{s_{1}^{\prime}}^{t}(t-s)^{-\frac{1}{2}} \mathbb{I} v^{(n-1)} \mathbb{I} \mathrm{d} s \\
& \leq\left(t-\bar{s}_{1}^{\prime}\right)^{-\frac{1}{4}} C_{1}\left(r, t-s_{1}^{\prime}\right) .
\end{aligned}
$$

For $k=2$,

$$
\begin{aligned}
D^{2} u^{(n)}(t, x)= & D^{2} T\left(t-s_{2}^{\prime}\right)\left(u^{(n)}\left(s_{2}^{\prime}, x\right)-\bar{u}\right)-\int_{s_{2}^{\prime}}^{t} D \Delta T(t-s) D f\left(u^{(n-1)}(s)\right) \mathrm{d} s \\
& -\int_{s_{2}^{\prime}}^{t} D^{2} T(t-s) \nabla \cdot g\left(u^{(n-1)}\right) \mathrm{d} s .
\end{aligned}
$$

Then, applying Hausdorff-Young's inequality and (3.12) with $k=1$ that

$$
\begin{aligned}
& \mathbb{I} D^{2} u^{(n)} \mathbb{I} \leq c_{1,2}\left(t-s_{2}^{\prime}\right)^{-\frac{1}{2}} \mathscr{I} u^{(n)} \mathbb{I}+b c_{1,3} \int_{s_{2}^{\prime}}^{t}(t-s)^{-\frac{3}{4}}\left(s-s_{1}^{\prime}\right)^{-\frac{1}{4}} C_{1}\left(r, s-s_{1}^{\prime}\right) \mathrm{d} s \\
& +b c_{1,2} \int_{s_{2}^{\prime}}^{t}(t-s)^{-\frac{1}{2}}\left(s-s_{1}^{\prime}\right)^{-\frac{1}{4}} C_{1}\left(r, s-s_{1}^{\prime}\right) \mathrm{d} s \\
& \leq\left(t-s_{2}^{\prime}\right)^{-\frac{1}{2}} C_{2}\left(r, s_{2}^{\prime}-s_{1}^{\prime}, t-s_{2}^{\prime}\right),
\end{aligned}
$$

which implies that (3.12) is true for $k=2$ and $n \geq 1$. In addition, we suppose that (3.12) holds for $k \leq m-1$ for some $3 \leq m \leq L$, i.e.,

$$
\mathbb{I} D^{k} u^{(n)} \mathbb{I} \leq\left(t-s_{k}^{\prime}\right)^{-\frac{k}{4}} C_{k}\left(r, s_{k}^{\prime}-s_{1}^{\prime}, t-s_{k}^{\prime}\right), \ldots k=1,2, \ldots, m-1
$$


Combining (3.7), (3.12) and (3.14) together, applying Hausdorff-Young's inequality, we derive that

$$
\begin{aligned}
& \mathbb{I} D^{m} u^{(n)} \mathbb{I} \\
& \leq c_{1, m}\left(t-s_{m}^{\prime}\right)^{-\frac{m}{4}} \mathbb{I} v^{(n)} \mathbb{I}+b c_{1,3} \int_{s_{m}^{\prime}}^{t}(t-s)^{-\frac{3}{4}} \sum_{\sum_{i=1}^{m} i \beta_{i}=m} \Pi_{i=1}^{m-1} \mathbb{I}\left(D^{i} u^{(n-1)}(s)\right)^{\beta_{i}} \mathbb{I} \mathrm{d} s \\
& \quad+b c_{1,2} \int_{s_{m}^{\prime}}^{t}(t-s)^{-\frac{1}{2}} \sum_{\sum_{i=1}^{m} i \beta_{i}=m} \Pi_{i=1}^{m-1} \mathbb{I}\left(D^{i} u^{(n-1)}(s)\right)^{\beta_{i}} \mathbb{I} \mathrm{d} s \\
& \leq 2 r c_{1, m}\left(t-s_{m}^{\prime}\right)^{-\frac{m}{4}} \\
& \quad+b c_{1,3} \int_{s_{m}^{\prime}}^{t}(t-s)^{-\frac{3}{4}} C_{1}\left(r, s-s_{1}^{\prime}\right) \cdots C_{m-1}\left(r, s_{m-1}^{\prime}-s_{1}^{\prime}, s-s_{m-1}^{\prime}\right) \mathrm{d} s \\
& \quad+b c_{1,2} \int_{s_{m}^{\prime}}^{t}(t-s)^{-\frac{1}{2}} C_{1}\left(r, s-s_{1}^{\prime}\right) \cdots C_{m-1}\left(r, s_{m-1}^{\prime}-s_{1}^{\prime}, s-s_{m-1}^{\prime}\right) \mathrm{d} s \\
& \leq\left(t-s_{m}^{\prime}\right)^{-\frac{m}{4}} C_{m}\left(r, s_{m}^{\prime}-s_{1}^{\prime}, t-s_{m}^{\prime}\right),
\end{aligned}
$$

which implies that (3.12) is true for $k=m$ and $n \geq 1$. Consequently, by induction, (3.12) holds for $1 \leq k \leq L, n \geq 1$.

Having obtained (3.12), it is a routine matter to verify that for each $\varsigma>0, D^{k} u^{(n)}(t, x)$ converges uniformly to $D^{k} u(t, x)$ on $\left[\varsigma, t_{1}\right] \times \mathbb{R}$ for $k=1,2, \ldots, L-1$. Hence, we obtain $u(t, x) \in C^{1,4}\left(\left[\varsigma, t_{1}\right] \times \mathbb{R}\right)$ and since $\varsigma>0$ can be chosen sufficiently small, we get $u(t, x) \in C^{1,4}\left(\left(0, t_{1}\right] \times \mathbb{R}\right)$. Applying the above regularity result, we can show that the solution $u(t, x)$ is indeed a smooth solution to problem (1.1) on $\Pi_{t_{1}}$ and (3.2) is the direct consequence of (3.13). Therefore, we complete the proof.

In the following, we establish the certain $L^{\frac{N(l-1)}{3}}\left(\mathbb{R}^{N}, \mathbb{R}\right)$ estimates on $u(t, x)$ on the time interval on which the smooth solutions exist.

Lemma 3.2 Suppose that the solution $u(t, x)$ obtained in Lemma 3.1 has been extended up to time $T\left(T \geq t_{1}>0\right)$ and the smooth properties and the a priori estimate (3.1) [and hence (3.2)] are kept unchanged. Then, for any $0<s_{1}^{\prime}<\bar{s}_{1}^{\prime}<s_{2}^{\prime}<\bar{s}_{2}^{\prime}<\cdots<s_{k}^{\prime}<\bar{s}_{k}^{\prime}<t \leq T$, we have

$$
\begin{aligned}
& \left\|D^{\frac{3}{l-1}} u\right\|_{L}{ }_{L}^{\frac{N(l-1)}{3}} \\
& \quad \leq C\left(t-\bar{s}_{k}^{\prime}\right)^{-\frac{3}{(4(l-1)}}\left(1+\mathcal{M}_{k}\left(r, t-\bar{s}_{k}^{\prime}, \bar{s}_{k}^{\prime}-s_{k}^{\prime}, t-\bar{s}_{k}^{\prime}\right)\right)\left\|u\left(\bar{s}_{k}^{\prime}\right)-\bar{u}\right\|_{L} \frac{N(l-1)}{3},
\end{aligned}
$$

where $k=2,3, \ldots, N$ and $\mathcal{M}_{k}$ is a continuous increasing function of $t-\bar{s}_{k}^{\prime}$.

Proof Note that

$$
\begin{aligned}
D^{\frac{3}{l-1}} u(t, x)= & D^{\frac{3}{l-1}} T\left(t-\bar{s}_{k}^{\prime}\right)\left(u\left(\bar{s}_{k}^{\prime}, x\right)-\bar{u}\right)-\int_{\bar{s}_{k} 1^{\prime}}^{t} D^{\frac{3}{l-1}+1} T(t-s) D f(u(s)) \mathrm{d} s \\
& -\int_{\bar{s}_{1}^{\prime}}^{t} D^{\frac{3}{l-1}} T(t-s) D g(u(s)) \mathrm{d} s .
\end{aligned}
$$


Then,

$$
\begin{aligned}
& \left\|D^{\frac{3}{l-1}} u(t)\right\|_{L^{\frac{N(l-1)}{3}}} \\
& \leq C\left(t-\bar{s}_{k}^{\prime}\right)^{-\frac{3}{4(l-1)}}\left\|u\left(\bar{s}_{k}^{\prime}, x\right)-\bar{u}\right\|_{L^{\frac{N(l-1)}{3}}}+b C \int_{\bar{s}_{k}^{\prime}}^{t}(t-s)^{-\frac{l+2}{4(l-1)}}\|u(s)-\bar{u}\|_{L} \frac{N(l-1)}{3} \mathrm{~d} s \\
& +b C \int_{\bar{s}_{k}^{\prime}}^{t}(t-s)^{-\frac{3}{4(l-1)}}\|u(s)\|_{L} \frac{N(l-1)}{3} \mathrm{~d} s \\
& \leq C\left(t-\bar{s}_{k}^{\prime}\right)^{-\frac{3}{(4(l-1)}}\left(1+\mathcal{M}_{k}\left(r, t-\bar{s}_{k}^{\prime}, \bar{s}_{k}^{\prime}-s_{k}^{\prime}, t-\bar{s}_{k}^{\prime}\right)\right)\left\|u\left(\bar{s}_{k}^{\prime}\right)-\bar{u}\right\|_{L} \frac{N(l-1)}{3} .
\end{aligned}
$$

The proof of Lemma 3.2 is completed.

The following lemma plays an important role in extending the local solution step by step to a global one.

Lemma 3.3 Suppose that the assumptions listed in Lemma 3.2 are satisfied, then there exists a positive constant $C_{1}(r)$ depending only on $r$ such that

$$
\|u-\bar{u}\|_{L} \frac{N(l-1)}{3}+t^{\frac{3}{4}}\|u-\bar{u}\|_{L} \frac{N l(l-1)}{3} \leq C_{1}(r)\left\|u_{0}-\bar{u}\right\|_{L} \frac{N(l-1)}{3}, \quad 0 \leq t \leq T,
$$

where the constant $C_{1}(r)$ is independent of $T$.

Proof Let

$$
X=\left\{u(t, x): u(t, x)-\bar{u} \in C\left(0, T ; L^{\frac{N(l-1)}{3}}\right), t^{\frac{3}{4 l}}(u(t, x)-\bar{u}) \in C\left(0, T ; L^{\frac{N l(l-1)}{3}}\right)\right\},
$$

with its norm defined by

$$
\|u\|_{X}=\sup _{[0, T]}\left\{\|u\|_{L} \frac{N(l-1)}{3}+t^{\frac{3}{4 l}}\|u\|_{L} \frac{N l(l-1)}{3}\right\} .
$$

It then follows from (3.5) that

$$
\begin{aligned}
\|u-\bar{u}\|_{X} \leq & \| T(t)\left(u_{0}-\bar{u}\left\|_{X}+\right\| \int_{0}^{t} D^{2} T(t-s) f(u(s)) \mathrm{d} s \|_{X}\right. \\
& +\left\|\int_{0}^{t} D T(t-s) g(u(s)) \mathrm{d} s\right\|_{X}=I_{1}+I_{2}+I_{3} .
\end{aligned}
$$

Based on Hausdorff-Young's inequality, we deduce that

$$
\begin{aligned}
I_{1} & =\sup _{[0, T]}\left\{\left\|T(t)\left(u_{0}-\bar{u}\right)\right\|_{L^{\frac{N(l-1)}{3}}}+t^{\frac{3}{4 l}}\left\|T(t)\left(u_{0}-\bar{u}\right)\right\|_{\left.L^{\frac{N l(l-1)}{3}}\right\}}\right. \\
& \leq \sup _{[0, T]}\left\{\|k(t)\|_{L^{1}}\left\|u_{0}-\bar{u}\right\|_{L^{\frac{N(l-1)}{3}}}+t^{\frac{3}{4 l}}\|k(t)\|_{L} \frac{N l(l-1)}{3}\left\|u_{0}-\bar{u}\right\|_{L} \frac{N(l-1)}{3}\right\} \\
& \leq \sup _{[0, T]}\left\{\left(1+c_{l} t^{\frac{3}{4 l}-\frac{1}{4}\left(\frac{3}{N(l-1)}-\frac{3}{N l(l-1)}\right)}\right)\left\|u_{0}-\bar{u}\right\|_{L}{ }_{L}^{\frac{N(l-1)}{3}}\right\} \\
& \leq\left(1+c_{l}\right)\left\|u_{0}-\bar{u}\right\|_{L} \frac{N(l-1)}{3} .
\end{aligned}
$$


For $I_{3}$, by employing a similar argument, we derive that

$$
\begin{aligned}
I_{3} \leq & \sup _{[0, T]}\left\{\int_{0}^{t}\|D T(t-s) g(u(s))\|_{L} \frac{N(l-1)}{3} \mathrm{~d} s+t^{\frac{3}{4 l}} \int_{0}^{t}\|D T(t-s) g(u(s))\|_{L} \frac{N l(l-1)}{3} \mathrm{~d} s\right\} \\
\leq & C_{2} \sup _{[0, T]}\left(\int_{0}^{t}(t-s)^{-\frac{1}{4}}\|u(s)-\bar{u}\|^{l}{ }_{L}^{\frac{N l(l-1)}{3}}\right. \\
& \left.\quad+t^{\frac{3}{4 l}} \int_{0}^{t}(t-s)^{-\frac{1}{4}\left(\frac{3}{N(l-1)}-\frac{3}{N l(l-1)}\right)-\frac{1}{4}}\|u(s)-\bar{u}\|_{L^{\frac{N l(l-1)}{3}}}^{l} \mathrm{~d} s\right) \\
\leq & C_{2} \sup _{[0, T]}\left(\int_{0}^{t}(t-s)^{-\frac{1}{4}} s^{-\frac{3}{4}} \mathrm{~d} s+t^{\frac{3}{4 l}} \int_{0}^{t}(t-s)^{-\frac{1}{4}\left(\frac{3}{N(l-1)}-\frac{3}{N l(l-1)}\right)-\frac{1}{4}} s^{-\frac{3}{4}} \mathrm{~d} s\right) \\
& \|u(s)-\bar{u}\|_{X}^{l} \leq C_{3}\|u-\bar{u}\|_{X}^{l} .
\end{aligned}
$$

As to $I_{2}$, note that $p=\frac{2 l+1}{3}$, we have

$$
\begin{aligned}
I_{2} \leq & \sup _{[0, T]}\left\{\int_{0}^{t}\left\|D^{2} T(t-s) f(u(s))\right\|_{L} \frac{N(l-1)}{3} \mathrm{~d} s+t^{\frac{3}{4 l}} \int_{0}^{t}\left\|D^{2} T(t-s) f(u(s))\right\|_{L} \frac{N l(l-1)}{3} \mathrm{~d} s\right\} \\
\leq & C_{4} \sup _{[0, T]}\left(\int_{0}^{t}(t-s)^{-\frac{1}{2}}\|u(s)-\bar{u}\|^{p}{ }^{\frac{N p(l-1)}{3}}\right. \\
& \left.+t^{\frac{3}{4 l}} \int_{0}^{t}(t-s)^{-\frac{1}{4}\left(\frac{3}{N(l-1)}-\frac{3}{N l(l-1)}\right)-\frac{1}{2}}\|u(s)-\bar{u}\|^{p}{ }^{\frac{N p(l-1)}{3}} \mathrm{~d} s\right) \\
\leq & C_{4} \sup _{[0, T]}\left(\int_{0}^{t}(t-s)^{-\frac{1}{2}}\|u(s)-\bar{u}\|^{\frac{l-p}{(l-1)}}\|u(s)-\bar{u}\|^{\frac{p l-l}{l-1}}{ }^{\frac{N l(l-1)}{3}}\right. \\
& \left.+t^{\frac{3}{4 l}} \int_{0}^{t}(t-s)^{-\frac{1}{4}\left(\frac{3}{N(l-1)}-\frac{3}{N l(l-1)}\right)-\frac{1}{2}}\|u(s)-\bar{u}\|^{\frac{l-p}{(l-1)}}\left\|{ }^{\frac{N(l-1)}{3}}\right\| u(s)-\bar{u} \|^{\frac{p l-l}{l-1}}{ }^{\frac{N l(l-1)}{3}} \mathrm{~d} s\right) \\
\leq & C_{4} \sup _{[0, T]}\left(\int_{0}^{t}(t-s)^{-\frac{1}{2}} s^{-\frac{1}{2}} \mathrm{~d} s+t^{\frac{3}{4 l}} \int_{0}^{t}(t-s)^{-\frac{1}{4}\left(\frac{3}{N(l-1)}-\frac{3}{N l(l-1)}\right)-\frac{1}{2}} s^{-\frac{1}{2}} \mathrm{~d} s\right) \\
& \times\|u(s)-\bar{u}\|^{\frac{l-p}{(l-1)}}\|u(s)-\bar{u}\|^{\frac{p-1-l}{l-1}}{ }^{\frac{N l(l-1)}{3}} \\
\leq & C_{4} \sup _{[0, T]}\left(\int_{0}^{t}(t-s)^{-\frac{1}{2}} s^{-\frac{1}{2}} \mathrm{~d} s+t^{\frac{3}{4 l}} \int_{0}^{t}(t-s)^{-\frac{1}{4}\left(\frac{3}{N(l-1)}-\frac{3}{N l(l-1)}\right)-\frac{1}{2}} s^{-\frac{1}{2}} \mathrm{~d} s\right) \\
& \|u(s)-\bar{u}\|_{X}^{l} \leq C_{5}\|u-\bar{u}\|_{X}^{l} \cdot
\end{aligned}
$$

Summing up, we immediately conclude

$$
\|u-\bar{u}\|_{X} \leq\left(1+c_{l}\right)\|u-\bar{u}\|_{L} \frac{N(l-1)}{3}+C\|u-\bar{u}\|_{X}^{l} .
$$

If we suppose that $\left\|u_{0}-\bar{u}\right\|_{L} \frac{N(l-1)}{3}$ is sufficiently small, applying Strauss's inequality, we can get (3.16) immediately. The proof is completed.

Now, we prove Theorem 1.1.

Proof of Theorem 1.1 Let $\beta$ be a sufficiently small positive constant. Choose $0<s_{1}<\overline{s_{1}}<$ $s_{2}<\bar{s}_{2}<s_{3}<\cdots<s_{N}<\bar{s}_{N} \leq T$ sufficiently small such that $\bar{s}_{N} \leq t_{1}$ and

$$
t_{1}-\bar{s}_{1}=\bar{s}_{i}-s_{i}=s_{i}=\bar{s}_{i-1}=\bar{s}_{1}=s_{1}=\beta, \quad i=2,3, \ldots, N .
$$


By (3.15) and (3.16), we have

$$
\left\{\begin{array}{l}
\|u(t)-\bar{u}\|_{L}{ }_{L}^{\frac{N(l-1)}{3}} \leq C_{1}(r)\left\|u_{0}-\bar{u}\right\|_{L}{ }_{L}^{\frac{N(l-1)}{3}}, 0 \leq t \leq t_{1}, \\
\left\|u\left(t_{1}\right)-\bar{u}\right\|_{W \frac{3}{l-1}, \frac{N(l-1)}{3}} \leq C_{7}\left(\beta, r, t_{1}\right) \sup _{\left[0, t_{1}\right]}\|u(t)-\bar{u}\|_{L} \frac{N(l-1)}{3} .
\end{array}\right.
$$

Assume that $\mathfrak{C}$ is the constant in Sobolev's embedding

$$
\|u(t)-\bar{u}\|_{L^{\infty}} \leq \mathfrak{C}\|u(t)-\bar{u}\|_{W \frac{3}{l-1}, \frac{N(l-1)}{3}},
$$

if we choose $\left\|u_{0}-\bar{u}\right\|_{L^{1}}$ sufficiently small such that

$$
\mathfrak{C} C_{1}(r) C_{7}\left(\beta, r, t_{1}\right)\left\|u_{0}-\bar{u}\right\|_{L} \frac{N(l-1)}{3} \leq\left\|u_{0}-\bar{u}\right\|_{L^{\infty}},
$$

then

$$
\begin{aligned}
& \left\|u\left(t_{1}\right)-\bar{u}\right\|_{L^{\infty}} \leq \mathfrak{C}\left\|u\left(t_{1}\right)-\bar{u}\right\|_{W \frac{3}{l-1}, \frac{N(l-1)}{3}} \leq \mathfrak{C} C_{7}\left(\beta, r, t_{1}\right) \sup _{\left[0, t_{1}\right]}\|u(t)-\bar{u}\|_{L^{\frac{N(l-1)}{3}}} \\
& \leq \mathfrak{C} C_{1}(r) C_{7}\left(\beta, r, t_{1}\right)\left\|u_{0}-\bar{u}\right\|_{L^{\frac{N(l-1)}{3}}} \leq\left\|u_{0}-\bar{u}\right\|_{L^{\infty}} \leq r .
\end{aligned}
$$

Follows from Lemmas 3.1 and 3.3, the solution $u(t, x)$ can be extended up to $2 t_{1}$ and satisfies

$$
\left\{\begin{array}{l}
\|u(t)-\bar{u}\|_{L^{\infty}} \leq 2 r, \quad 0 \leq t \leq 2 t_{1}, \\
\|u(t)-\bar{u}\|_{L^{\frac{N(l-1)}{3}} \leq C_{1}(r)\left\|u_{0}-\bar{u}\right\|_{L^{\frac{N(l-1)}{3}}}, \quad 0 \leq t \leq 2 t_{1} .}
\end{array}\right.
$$

Take $t=2 t_{1}, s_{1}^{\prime}=s_{1}+t_{1}$ and $\bar{s}_{1}^{\prime}=\bar{s}_{1}+t_{1}$ in (3.15) and (3.16), we derive that

$$
\left\|u\left(2 t_{1}\right)-\bar{u}\right\|_{W \frac{3}{l-1}, \frac{N(l-1)}{3}} \leq C_{7}\left(\beta, r, t_{1}\right) \sup _{\left[0,2 t_{1}\right]}\|u(t)-\bar{u}\|_{L} \frac{N(l-1)}{3} .
$$

Now, assume that $u(t, x)$ has been defined up to $k t_{1}$ for some $k \in \mathbb{Z}_{+}$such that

$$
\left\{\begin{array}{l}
\|u(t)-\bar{u}\|_{L^{\infty}} \leq 2 r, \quad 0 \leq t \leq k t_{1}, \\
\|u(t)-\bar{u}\|_{L^{\frac{N(l-1)}{3}} \leq C_{1}(r)\left\|u_{0}-\bar{u}\right\|_{L} \frac{N(l-1)}{3}}, \quad 0 \leq t \leq k t_{1} .
\end{array}\right.
$$

Take $t=k t_{1}, s_{1}^{\prime}=s_{1}+(k-1) t_{1}$ and $\bar{s}_{1}^{\prime}=\bar{s}_{1}+(k-1) t_{1}$ in (3.15) and (3.16), we derive that

$$
\left\|u\left(k t_{1}\right)-\bar{u}\right\|_{W \frac{3}{l-1}, \frac{N(l-1)}{3}} \leq C_{7}\left(\beta, r, t_{1}\right) \sup _{\left[0, k t_{1}\right]}\|u(t)-\bar{u}\|_{L}{ }_{L}^{\frac{N(l-1)}{3}} .
$$

It then follows from (3.19), (3.22) and (3.23) that

$$
\begin{aligned}
\left\|u\left(k t_{1}\right)-\bar{u}\right\|_{L^{\infty}} & \leq \mathfrak{C}\left\|u\left(k t_{1}\right)-\bar{u}\right\|_{W^{\frac{3}{l-1}}, \frac{N(l-1)}{3}} \leq \mathfrak{C} C_{7}\left(\beta, r, t_{1}\right) \sup _{\left[0, t_{1}\right]}\|u(t)-\bar{u}\|_{L^{\frac{N(l-1)}{3}}} \\
& \leq \mathfrak{C} C_{1}(r) C_{7}\left(\beta, r, t_{1}\right)\left\|u_{0}-\bar{u}\right\|_{L^{\frac{N(l-1)}{3}}} \leq\left\|u_{0}-\bar{u}\right\|_{L^{\infty}} \leq r .
\end{aligned}
$$

Follows from Lemmas 3.1 and 3.3 again, the solution $u(t, x)$ can be extended up to $(k+1) t_{1}$ and satisfies

$$
\left\{\begin{array}{l}
\|u(t)-\bar{u}\|_{L^{\infty}} \leq 2 r, \quad 0 \leq t \leq(k+1) t_{1}, \\
\|u(t)-\bar{u}\|_{L} \frac{N(l-1)}{3} \leq C_{1}(r)\left\|u_{0}-\bar{u}\right\|_{L}{ }^{\frac{N(l-1)}{3}}, \quad 0 \leq t \leq(k+1) t_{1} .
\end{array}\right.
$$

Proceeding inductively, we thus establish the existence of solution $u(x, t)$ in all $t>0$. The proof is completed. 


\section{The brief proof of Theorem 1.2}

In this section, we give a brief proof of Theorem 1.2.

Similar to the proof of Theorem 1.1, we first consider the existence and uniqueness of local smooth solutions for problem (1.5).

By using the same method as the proof of Lemma 3.1, we have

Lemma 4.1 Suppose that $\max _{u \in \bar{B}(\bar{u}, 2 r)} \sum_{k=1}^{L}\left|D^{k} f(u)\right|=b$ and the conditions listed in Theorem 1.2 are satisfied. Then, there exists a unique local solution $u(x, t)$ on the strip $\Pi_{t_{1}}:=\left\{(x, t): x \in \mathbb{R}^{N}, 0<t \leq t_{1}\right\}$ such that

$$
\|u(x, t)-\bar{u}\|_{L^{\infty}} \leq 2 r, \quad 0 \leq t \leq t_{1},
$$

where $t_{1}=\min \left\{1, \frac{1}{64 b^{2} c_{1,2}^{2}}\right\}$. In addition, assume that $C_{k}$ is a continuous increasing function of $t-s_{k}^{\prime}$, then for each $0<s_{1}^{\prime}<s_{2}^{\prime}<\cdots<s_{7}^{\prime}<t \leq t_{1}$, we have

$$
\left\|D^{k} u(t, x)\right\|_{L^{\infty}} \leq\left(t-s_{k}^{\prime}\right)^{-\frac{k}{4}} C_{k}\left(r, s_{k}^{\prime}-s_{1}^{\prime}, t-s_{k}^{\prime}\right), \quad k=1,2, \ldots L .
$$

It is worth pointing that the integro-differential equation of (1.5) is

$$
u(t, x)=\int k(t, x-y) u_{0} \mathrm{~d} y-\int_{0}^{t} \mathrm{~d} s \int \Delta k(x-y, t-s) f(u(s, y)) \mathrm{d} y .
$$

and the main difference between the proof of Lemma 3.1 and the proof of Lemma 4.1 is the choice of $t_{1}$. In Lemma 4.1, we assume that $t_{1}=\min \left\{1, \frac{1}{64 b^{2} c_{1,2}^{2}}\right\}$ whereas $t_{1}=$ $\min \left\{1, \frac{1}{64 b^{2} c_{1,2}^{2}},\left(\frac{3}{16 b c_{1,1}}\right)^{\frac{4}{3}}\right\}$ in Lemma 3.1.

The certain $L^{\frac{N(l-1)}{2}}\left(\mathbb{R}^{N}, \mathbb{R}\right)$ estimates on $u(t, x)$ on the time interval can be established similar to Lemma 3.2.

Lemma 4.2 Suppose that the solution $u(t, x)$ obtained in Lemma 4.1 has been extended up to time $T\left(T \geq t_{1}>0\right)$ and the smooth properties and the a priori estimate (4.1) (and hence (4.2)) are kept unchanged. Then, for any $0<s_{1}^{\prime}<\bar{s}_{1}^{\prime}<s_{2}^{\prime}<\bar{s}_{2}^{\prime}<\cdots<s_{k}^{\prime}<\bar{s}_{k}^{\prime}<t \leq T$,

$$
\begin{aligned}
& \left\|D^{\frac{2}{l-1}} u\right\|_{L}{ }^{\frac{N(l-1)}{2}} \\
& \leq C\left(t-\bar{s}_{k}^{\prime}\right)^{-\frac{1}{(2(l-1)}}\left(1+\mathcal{M}_{k}\left(r, t-\bar{s}_{k}^{\prime}, \bar{s}_{k}^{\prime}-s_{k}^{\prime}, t-\bar{s}_{k}^{\prime}\right)\right)\left\|u\left(\bar{s}_{k}^{\prime}\right)-\bar{u}\right\|_{L} \frac{N(l-1)}{3} .
\end{aligned}
$$

where $k=2,3, \ldots, N$ and $\mathcal{M}_{k}$ is a continuous increasing function of $t-\bar{s}_{k}^{\prime}$.

We also have the following lemma, which is concerned with the time-independent $L^{\frac{N(l-1)}{2}}(\mathbb{R}, \mathbb{R})$ a priori estimate on the solution $u(t, x)$ of problem (1.5).

Lemma 4.3 Suppose that the assumptions listed in Lemma 4.2 are satisfied, then there exists a positive constant $C_{2}(r)$ depending only on $r$ such that

$$
\|u-\bar{u}\|_{L} \frac{N(l-1)}{2}+t^{\frac{1}{2 l}}\|u-\bar{u}\|_{L} \frac{N l(l-1)}{2} \leq C_{2}(r)\left\|u_{0}-\bar{u}\right\|_{L} \frac{N(l-1)}{2}, \quad 0 \leq t \leq T,
$$

where the constant $C_{1}(r)$ is independent of $T$. 
Proof Let

$X=\left\{u(t, x): u(t, x)-\bar{u} \in C\left(0, T ; L^{\frac{N(l-1)}{2}}\right), t^{\frac{1}{2 l}}(u(t, x)-\bar{u}) \in C\left(0, T ; L^{\frac{N l(l-1)}{2}}\right)\right\}$,

with its norm defined by

$$
\|u\|_{X}=\sup _{[0, T]}\left\{\|u\|_{L} \frac{N(l-1)}{2}+t^{\frac{1}{2 l}}\|u\|_{L} \frac{N l(l-1)}{2}\right\} .
$$

Then,

$$
\|u-\bar{u}\|_{X} \leq \| T(t)\left(u_{0}-\bar{u}\left\|_{X}+\right\| \int_{0}^{t} D^{2} T(t-s) f(u(s)) \mathrm{d} s \|_{X}=I_{1}+I_{2} .\right.
$$

Note that

$$
\begin{aligned}
& I_{1}=\sup _{[0, T]}\left\{\left\|T(t)\left(u_{0}-\bar{u}\right)\right\|_{L} \frac{N(l-1)}{2}+t^{\frac{1}{2 l}}\left\|T(t)\left(u_{0}-\bar{u}\right)\right\|_{L^{\frac{N l(l-1)}{2}}}\right\} \\
& \leq \sup _{[0, T]}\left\{\|k(t)\|_{L^{1}}\left\|u_{0}-\bar{u}\right\|_{L^{\frac{N(l-1)}{2}}}+t^{\frac{1}{2 l}}\|k(t)\|_{L} \frac{N l(l-1)}{2}\left\|u_{0}-\bar{u}\right\|_{L}{ }_{L}^{\frac{N(l-1)}{2}}\right\} \\
& \leq \sup _{[0, T]}\left\{\left(1+c_{l} t^{\frac{1}{2 l}-\frac{N}{4}\left(\frac{2}{N(l-1)}-\frac{2}{N l(l-1)}\right)}\right)\left\|u_{0}-\bar{u}\right\|_{L} \frac{N(l-1)}{3}\right\} \leq\left(1+c_{l}\right)\left\|u_{0}-\bar{u}\right\|_{L}{ }_{L}^{\frac{N(l-1)}{2}} .
\end{aligned}
$$

and

$$
\begin{aligned}
& I_{2} \leq \sup _{[0, T]}\left\{\int_{0}^{t}\left\|D^{2} T(t-s) f(u(s))\right\|_{L^{\frac{N(l-1)}{2}}} \mathrm{~d} s+t^{\frac{1}{2 l}} \int_{0}^{t}\left\|D^{2} T(t-s) f(u(s))\right\|_{L^{\frac{N l(l-1)}{2}}} \mathrm{~d} s\right\} \\
& \leq C_{2} \sup _{[0, T]}\left(\int_{0}^{t}(t-s)^{-\frac{1}{2}}\|u(s)-\bar{u}\|_{L}^{l} \frac{N l(l-1)}{2}\right. \\
& \left.+t^{\frac{1}{2 l}} \int_{0}^{t}(t-s)^{-\frac{N}{4}\left(\frac{2}{N(l-1)}-\frac{2}{N l(l-1)}\right)-\frac{1}{2}}\|u(s)-\bar{u}\|_{L}^{l}{ }_{L}^{\frac{N l(l-1)}{2}} \mathrm{~d} s\right) \\
& \leq C_{2} \sup _{[0, T]}\left(\int_{0}^{t}(t-s)^{-\frac{1}{2}} s^{-\frac{1}{2}} \mathrm{~d} s+t^{\frac{1}{2 l}} \int_{0}^{t}(t-s)^{-\frac{N}{4}\left(\frac{2}{N(l-1)}-\frac{2}{N l(l-1)}\right)-\frac{1}{2}} s^{-\frac{1}{2}} \mathrm{~d} s\right) \\
& \|u(s)-\bar{u}\|_{X}^{l} \leq C_{3}\|u-\bar{u}\|_{X}^{l} .
\end{aligned}
$$

Summing up, we immediately conclude

$$
\|u-\bar{u}\|_{X} \leq\left(1+c_{l}\right)\|u-\bar{u}\|_{L} \frac{N(l-1)}{2}+C\|u-\bar{u}\|_{X}^{l} .
$$

If we suppose that $\left\|u_{0}-\bar{u}\right\|_{L} \frac{N(l-1)}{2}$ is sufficiently small, applying Strauss's inequality, we can get (4.5) immediately.

The proof of Theorem 1.2 is in the following.

Proof of Theorem 1.2 With the above preparations in hand, proceeding inductively, we thus establish the existence of the solution $u(x, t)$ for problem (1.5) in all $t>0$. Hence, the proof of Theorem 1.1 is completed.

Acknowledgements This paper is supported by the Natural Science Foundation of China (Grant No. 11401258), Natural Science Foundation of Jiangsu Province (Grant No. BK20140130) and China Postdoctoral Science Foundation (Grant No. 2015M581689). 


\section{References}

1. Adams, R.A., Folurnier, J.J.F.: Sobolev Spaces, 2nd edn. Elsevier, Amsterdam (2003)

2. Akagi, G., Schimperna, G., Segatti, A.: Fractional Cahn-Hilliard, Allen-Cahn and porous medium equations. J. Differ. Eqs. 261, 2935-2985 (2016)

3. Blomker, D., Maier-Paape, S., Wanner, T.: Spinodal decomposition for the Cahn-Hilliard-Cook equation. Commun. Math. Phys. 223, 553-582 (2001)

4. Bonfoh, A., Miranville, A.: On Cahn-Hilliard-Gurtin equations. In: Proceedings of the Third World Congress of Nonlinear Analysis, Part 5 (Catania, 2000), Nonlinear Analysts, vol. 47, pp. 3455-3466 (2001)

5. Bonfoh, A.: Finite-dimensional attractor for the viscous Cahn-Hilliard equation in an unbounded domain. Q. Appl. Math. 64, 93C104 (2006)

6. Bosia, S., Conti, M., Grasselli, M.: On the Cahn-Hilliard-Brinkman system. Commun. Math. Sci. 13, 1541-1567 (2015)

7. Cahn, J.W., Hilliard, J.E.: Free energy of a nonuniform system. I. Interfacial free energy. J. Chem. Phys. 28, 258-267 (1958)

8. Dai, S., Qiang, D.: Weak solutions for the Cahn-Hilliard equation with degenerate mobility. Arch. Rational Mech. Anal. 219, 1161-1184 (2016)

9. Della Porta, F., Grasselli, M.: Convective nonlocal Cahn-Hilliard equations with reaction terms. Discrete Contin. Dyn. Syst. Ser. B 20, 1529-1553 (2015)

10. Di Nezza, E., Palatucci, G., Valdinoci, E.: Hitchhiker's guide to the fractional Sobolev spaces. Bull. Sci. Math. 136, 521-573 (2012)

11. Ding, X.X., Wang, J.H.: Global solution for a semilinear parabolic system. Acta Math. Sci. 3, 397-414 (1983)

12. Dlotko, T.: Global attractor for the Cahn-Hilliard equation in $H^{2}$ and $H^{3}$. J. Differ. Eqs. 113, 381-393 (1994)

13. Dlotko, T., Kania, M.B., Sun, C.: Analysis of the viscous Cahn-Hilliard equation in $R^{N}$. J. Differ. Eqs. 252, 2771-2791 (2012)

14. Eden, A., Kalantarov, V.K.: 3D convective Cahn-Hilliard equation. Commu. Pure Appl. Anal. 6, 10751086 (2007)

15. Eden, A., Kalantarov, V.K.: The convective Cahn-Hilliard equation. Appl. Math. Lett. 20, 455-461 (2007)

16. Elliott, C.M., Zheng, S.M.: On the Cahn-Hilliard equation. Arch. Rational Mech. Anal. 96, 339-357 (1986)

17. Friedman, A.: Partial Differential Equations. Holt, Reinhart and Winston, New York (1969)

18. Gilardi, G., Miranville, A., Schimperna, G.: On the Cahn-Hilliard equation with irregular potentials and dynamic boundary conditions. Commun. Pure Appl. Anal. 8, 881-912 (2009)

19. Golovin, A.A., Davis, S.H., Nepomnyashchy, A.A.: A convective Cahn-Hilliard model for the formation of facets and corners in crystal growth. Phys. D 122, 202-230 (1998)

20. Hoff, D., Smooler, J.A.: Solutions in the large for certain nonlinear parabolic systems. Ann. Inst. H. Poincaré Anal. Non Linéaire 2, 213-235 (1985)

21. Hoff, D., Smooler, J.A.: Global existence for parabolic conservation laws. J. Differ. Eqs. 68, 210-220 (1987)

22. Injrou, S., Pierre, M.: Error estimates for a finite element discretization of the Cahn-Hilliard-Gurtin equations. Adv. Differ. Eqs. 15, 1161-1192 (2010)

23. Kwasnicki, M.: Ten equivalent definitions of the fractional Laplace operator. Fract. Calc. Appl. Anal. 20, 7-51 (2017)

24. Liu, S., Wang, F., Zhao, H.: Global existence and asymptotics of solutions of the Cahn-Hilliard equation. J. Differ. Eqs. 238, 426-469 (2007)

25. Novick-Cohen, A.: On Cahn-Hilliard type equations. Nonlinear Anal. 15, 797-814 (1990)

26. Novick-Cohen, A., Segel, L.A.: Nonlinear aspects of the Cahn-Hilliard equation. Phys. D 10, $277-298$ (1984)

27. Robinson, J.C., Rodrigo, J.L., Sadowski, W.: The Three-Dimensional Navier-Stokes Equations, Cambridge Studies in Advanced Mathematics. Cambridge University Press, Cambridge (2016)

28. Rocca, E., Sprekels, J.: Optimal distributed control of a nonlocal convective Cahn-Hilliard equation by the velocity in three dimensions. SIAM J. Control Optim. 53, 1654-1680 (2015)

29. Strauss, W.A.: Decay and asymptotic for $u_{t t}-\Delta u=F(u)$. J. Funct. Anal. 2, 409-457 (1968)

30. Temam, R.: Infinite Dimensional Dynamical Systems in Mechanics and Physics, Applied Mathematical Sciences, vol. 68. Springer, New York (1988)

31. Wang, X., Zhang, Z.: Well-posedness of the Hele-Shaw-Cahn-Hilliard system. Ann. Inst. H. Poincaré Anal. Non Linéaire 30, 367-384 (2013) 
32. Watson, S.J., Otto, F., Rubinstein, B.Y., Davis, S.H.: Coarsening dynamics of the convective Cahn-Hilliard equation. Phys. D 178, 127-148 (2003)

33. Yin, J.: On the existence of nonnegative continuous solutions of the Cahn-Hilliard equation. J. Differ. Eqs. 97, 310-327 (1992)

34. Zarks, M.A., Podolny, A., Nepomnyashchy, A.A., Golovin, A.A.: Periodic stationary patterns governed by a convective Cahn-Hilliard equation. SIAM J. Appl. Math. 66, 700-720 (2005)

35. Zhao, X., Liu, C.: Optimal control for the convective Cahn-Hilliard equation in 2D case. Appl. Math. Optim. 70, 61-82 (2014) 